Kragujevac Journal of Mathematics

Volume 40(2) (2016), Pages 146-164.

\title{
CHEN-LIKE INEQUALITIES ON LIGHTLIKE HYPERSURFACE OF A LORENTZIAN PRODUCT MANIFOLD WITH QUARTER-SYMMETRIC NONMETRIC CONNECTION
}

\author{
NERGIZ (ÖNEN) POYRAZ ${ }^{1}$ AND EROL YAŞAR ${ }^{2}$
}

\begin{abstract}
In this paper, we introduce $k$-Ricci curvature and $k$-scalar curvature on lightlike hypersurface of a Lorentzian product manifold with quarter-symmetric nonmetric connection. Using these curvatures, we establish some Chen-type inequalities for lighlike hypersurface of a Lorentzian product manifold with quarter-symmetric nonmetric connection. Considering the equality case, we obtain some results.
\end{abstract}

\section{INTRODUCTION}

In [16], Golab introduced the idea of a quarter-symmetric linear connections in a differential manifold. Later, the properties of Riemannian manifolds with quartersymmetric metric (nonmetric) connection have been studied by some authours [19,24].

Warped products were first defined by Bishop and O'Neill in [6]. In [2], Atçeken and Kılıç introduced semi-invariant lightlike submanifolds of a semi-Riemannian product manifold. In [20], Kılıç and Oğuzhan considered lightlike hypersurfaces with respect to a quarter-symmetric nonmetric connection which is determined by the product structure. They also gave some equivalent conditions for integrability of disributions with respect to the Levi-Civita connection of semi-Riemannian manifold and the quarter-symmetric nonmetric connection, and obtained some results.

In 1993, B. Y. Chen [9] introduced a new Riemannian invariant for a Riemannian manifold $M$ as follows:

$$
\delta_{M}(p)=\tau(p)-\inf (K)(p)
$$

Key words and phrases. Lightlike hypersurface, Lorentzian manifold, Quarter-symmetric nonmetric connection, Curvature, Ricci curvature.

2010 Mathematics Subject Classification. Primary: 53B05, 53B15, 53C40, 53C042, 53C50.

Received: May 11, 2015.

Accepted: January 6, 2016. 
where $\tau(p)$ is scalar curvature of $M$ and

$$
\inf (K)(p)=\inf \left\{K(\Pi): K(\Pi) \text { is a plane section of } T_{p} M\right\} .
$$

In [9], B. Chen established a sharp inequality for submanifolds in a real space form involving $\delta_{M}$ and the main extrinsic invariant, namely the squared mean curvature.

Afterwards, B. Y. Chen and some geometers studied similar problems for nondegenerate submanifolds of different spaces such as in $[8,9,17,28]$. Later, Mihai and Özgür in [22] proved Chen inequalities for submanifolds of real space forms endowed with a semi-symmetric metric connection.

In degenerate submanifolds, M. Gülbahar, E. Kılıç and S. Keleş introduced $k$-Ricci curvature, $k$-scalar curvature, $k$-degenerate Ricci curvature, $k$-degenerate scalar curvature and they established some inequalities that characterize lightlike hypersurface of a Lorentzian manifold in [17]. After, they established some inequalities involving $k$-Ricci curvature, $k$-scalar curvature, the screen scalar curvature on a screen homothetic lightlike hypersurface of a Lorentzian manifold and they computed Chen-Ricci inequality and Chen inequality on a screen homothetic lightlike hypersurface of a Lorentzian manifold in [18].

In this paper, we study Chen-type inequalities for screen homothetic lightlike hypersurface of a real product space form $\widetilde{M}(c)=M_{1}\left(c_{1}\right) \times M_{2}\left(c_{2}\right)$ of constant sectional curvature $c$, endowed with quarter-symmetric nonmetric connection. Considering these inequalities, we obtain the relation between Ricci curvature and scalar curvature endowed with the quarter-symmetric nonmetric connection.

\section{PRELiminaries}

Let $M$ be a hypersurface of an $(n+1)$-dimensional, $n>1$, semi-Riemannian manifold $\widetilde{M}$ with semi-Riemannian metric $\widetilde{g}$ of index $1 \leq \nu \leq n$. We consider

$$
T_{x} M^{\perp}=\left\{Y_{x} \in T_{x} \widetilde{M} \mid \widetilde{g}_{x}\left(Y_{x}, X_{x}\right)=0, \quad \text { for all } X_{x} \in T_{x} M\right\},
$$

for any $x \in M$. Then we say that $M$ is a lightlike (null, degenerate) hypersurface of $\widetilde{M}$ or equivalently, the immersion

$$
i: M \rightarrow \widetilde{M}
$$

of $M$ in $\widetilde{M}$ is lightlike (null, degenerate) if $T_{x} M \cap T_{x} M^{\perp} \neq\{0\}$ at any $x \in M$. Henceforth we identify $i(M)$ with $M$ and we denote the differential $d i$, immersing a vector field $X$ in $M$ to a vector field $\phi X$ in $\widetilde{M}$, by $\phi$. Thus the induced metric tensor $g=\widetilde{g}_{\left.\right|_{M}}$ is defined by

$$
g(X, Y)=\widetilde{g}(\phi X, \phi Y), \quad \text { for all } X, Y \in \Gamma(T M) .
$$

An orthogonal complementary vector bundle of $T M^{\perp}$ in $T M$ is non-degenerate subbundle of $T M$ called the screen distribution on $M$ and denoted by $S(T M)$. We have the following splitting into orthogonal direct sum:

$$
T M=S(T M) \perp T M^{\perp} .
$$


The subbundle $S(T M)$ is non-degenerate, so is $S(T M)^{\perp}$, and the following holds:

$$
T \widetilde{M}=S(T M) \perp S(T M)^{\perp},
$$

where $S(T M)^{\perp}$ is the orthogonal complementary vector bundle to $S(T M)$ in $\left.T \widetilde{M}\right|_{M}$.

Let $\operatorname{tr}(T M)$ denote the complementary vector bundle of $T M^{\perp}$ in $S(T M)^{\perp}$. Then we have

$$
S(T M)^{\perp}=T M^{\perp} \oplus \operatorname{tr}(T M) .
$$

Let $\mathcal{U}$ be a coordinate neighbourhood in $M$ and $\xi$ be a basis of $\Gamma\left(\left.T M^{\perp}\right|_{\mathcal{U}}\right)$. Then there exists a basis $N$ of $\left.\operatorname{tr}(T M)\right|_{u}$ satisfying the following conditions:

$$
\widetilde{g}(N, \xi)=1
$$

and

$$
\widetilde{g}(N, N)=\widetilde{g}(W, N)=0, \quad \text { for all } W \in \Gamma\left(\left.S(T M)\right|_{u}\right) .
$$

The subbundle $\operatorname{tr}(T M)$ is called a lightlike transversal vector bundle of $M$. We note that $\operatorname{tr}(T M)$ is never orthogonal to $T M$. From (2.1), (2.2) and (2.3) we have

$$
\left.T \widetilde{M}\right|_{M}=S(T M) \perp\left(T M^{\perp} \oplus \operatorname{tr}(T M)\right)=T M \oplus \operatorname{tr}(T M) .
$$

Let $\stackrel{\circ}{\widetilde{\nabla}}$ be the Levi-Civita connection of $\widetilde{M}$ and $P$ be the projection morphism of $\Gamma(T M)$ on $\Gamma(S(T M))$. The Gauss and Weingarten formulas are given

$$
\begin{aligned}
\stackrel{\circ}{\nabla}_{X} Y & =\stackrel{\circ}{\nabla}_{X} Y+B(X, Y) N, \\
\stackrel{\circ}{\nabla}_{X} Y & =-\stackrel{\circ}{A}_{N} X+\omega(X) N, \\
\stackrel{\circ}{\nabla}_{X} P Y & =\stackrel{\circ}{\nabla}_{X} P Y+C(X, P Y) \xi, \\
& \stackrel{*}{\circ} \stackrel{\circ}{\nabla}_{X}=-\stackrel{\circ}{A}_{\xi} X-\omega(X) \xi,
\end{aligned}
$$

for any $X, Y \in \Gamma(T M)$, where $\stackrel{\circ}{\nabla}$ and $\stackrel{*}{\nabla}$ are the induced linear connection on $T M$ and $S(T M)$, respectively; $B$ and $C$ are the local second fundemental forms on $T M$

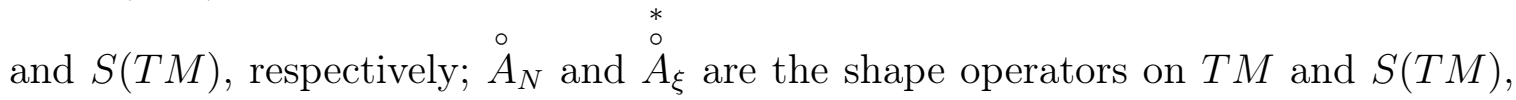
respectively; and $\omega$ is a 1 -form on $T M[14,15]$. Also, the local second fundamental forms $B$ and $C$ of $T M$ and $S(T M)$, respectively; are related to their shape operators $\stackrel{\circ}{A_{N}}$ and $\stackrel{*}{A_{\xi}}$ by

$$
\begin{aligned}
B(X, Y) & =g\left(\stackrel{*}{A_{\xi}} X, Y\right), \\
C(X, P Y) & =g\left(\stackrel{\circ}{A}_{N} X, P Y\right) .
\end{aligned}
$$


If $B=0$, then the lightlike hypsersurface $M$ is called totally geodesic in $\widetilde{M}$. A point $p \in M$ is said to be umbilical if

$$
B(X, Y)_{p}=H g_{p}(X, Y), \quad X, Y \in \Gamma\left(T_{p} M\right),
$$

where $H \in R$. The lightlike hypsersurface $M$ is called totally umbilical in $\widetilde{M}$ if every points of $M$ is umbilical [14].

The mean curvature $\mu$ of $M$ with respect to an orthonormal basis $\left\{e_{1}, \ldots, e_{n}\right\}$ of $\Gamma(S(T M))$ is defined in [5] as follows:

$$
\mu=\frac{1}{n} \operatorname{tr}(B)=\frac{1}{n} \sum_{i=1}^{n} \varepsilon_{i} B\left(e_{i}, e_{i}\right), \quad g\left(e_{i}, e_{i}\right)=\varepsilon_{i} .
$$

A Lightlike hypersurface $(M, g)$ of a semi-Riemannian manifold $(\widetilde{M}, \widetilde{g})$ is called screen locally conformal if the shape operators $\stackrel{\circ}{A_{N}}$ and $\stackrel{*}{\stackrel{*}{A}}$. of $M$ and $S(T M)$, respectively, are related by

$$
\stackrel{\circ}{A_{N}}=\stackrel{*}{\varphi} \stackrel{\circ}{A}_{\xi}
$$

where $\varphi$ is a non-vanishing smooth function on a neighbourhood $\mathcal{U}$ on $M$. In particular, $M$ is called screen homothetic if $\varphi$ is non-zero constant [3].

We denote by $\stackrel{\circ}{\widetilde{R}}$ the curvature tensor of $\widetilde{M}$ with respect to Levi-Civita connection $\stackrel{\circ}{\widetilde{\nabla}}$ and by $\stackrel{\circ}{R}$ that of $M$ with respect to induced connection $\stackrel{\circ}{\nabla}$. Then the Gauss equations of $M$ is given by

$$
\begin{aligned}
\stackrel{\circ}{\widetilde{R}}(X, Y) Z= & \stackrel{\circ}{R}(X, Y) Z+A_{h(X, Z)} Y-A_{h(Y, Z)} X \\
& +\left(\nabla_{X} h\right)(Y, Z)-\left(\nabla_{Y} h\right)(X, Z),
\end{aligned}
$$

for $X, Y, Z, W \in \Gamma(T M)$.

Let $M$ be a two-dimensional non-degenerate plane. The number

$$
K_{i j}=\frac{g\left(R\left(e_{j}, e_{i}\right) e_{i}, e_{j}\right)}{g\left(e_{i}, e_{i}\right) g\left(e_{j}, e_{j}\right)-g\left(e_{i}, e_{j}\right)^{2}}
$$

is called the sectional curvature of the plane section spanned by $e_{i}$ and $e_{j}$ at $p \in M$ [15].

Let $p \in M$ and $\xi$ be null vector of $T_{p} M$. A plane $\Pi$ of $T_{p} M$ is said to be null plane if it contains $\xi$ and $e_{i}$ such that $g\left(\xi, e_{i}\right)=0$ and $g\left(e_{i}, e_{i}\right)=\varepsilon_{i}= \pm 1$. The null sectional curvature of $\Pi$ is given in [4] as follows

$$
K_{i}^{\text {null }}=\frac{g\left(R_{p}\left(e_{i}, \xi\right) \xi, e_{i}\right)}{g_{p}\left(e_{i}, e_{i}\right)} .
$$


The Ricci tensor $\widetilde{\text { Ric }}$ of $\widetilde{M}$ and the induced Ricci type tensor $R^{(0,2)}$ of $M$ are defined by

$$
\begin{aligned}
\widetilde{\operatorname{Ric}}(X, Y) & =\operatorname{trace}\{Z \rightarrow \widetilde{R}(Z, X) Y\}, \quad \text { for all } X, Y \in \Gamma(T \widetilde{M}), \\
R^{(0,2)}(X, Y) & =\operatorname{trace}\{Z \rightarrow R(Z, X) Y\}, \quad \text { for all } X, Y \in \Gamma(T M),
\end{aligned}
$$

where

$$
R^{(0,2)}(X, Y)=\sum_{i=1}^{n} \varepsilon_{i} g\left(R\left(e_{i}, X\right) Y, e_{i}\right)+g(R(\xi, X) Y, N)
$$

for the quasi-orthonormal frame $\left\{e_{1}, \ldots, e_{n}, \xi\right\}$ of $T_{p} M$.

If $M$ admits that an induced symmetric Ricci tensor Ric and Ricci tensor satisfy

$$
\operatorname{Ric}(X, Y)=k g(X, Y) \text {, }
$$

where $k$ is a constant, then $M$ is called an Einstein hypersurface [15].

\section{Lorentzian Product Manifolds}

In this section, we use the same notations and terminologies as in [20].

Let $\left(M_{1}, g_{1}\right)$ and $\left(M_{2}, g_{2}\right)$ be two $\left(m_{1}+1\right)$ and $\left(m_{2}+1\right)$ dimensional Lorentzian manifolds with constant indexes $q_{1}>0, q_{2}>0$, respectively, and $\widetilde{M}=\left(M_{1} \times M_{2}, \widetilde{g}\right)$ be $\left(m_{1}+m_{2}+2\right)$-dimensional differentiable manifold with a tensor field $F$ of type $(1,1)$ on $\widetilde{M}$ such that

$$
F^{2}=I
$$

Let $\pi: M_{1} \times M_{2} \rightarrow M_{1}$ and $\sigma: M_{1} \times M_{2} \rightarrow M_{2}$ be the projections which are given by $\pi(x, y)=x$ and $\sigma(x, y)=y$ for any $(x, y) \in M_{1} \times M_{2}$. Then $\widetilde{M}=M_{1} \times M_{2}$ is called an almost product manifold with almost product structure $F$. If we put

$$
\pi=\frac{1}{2}(I+F), \quad \sigma=\frac{1}{2}(I-F)
$$

then we have

$$
\pi^{2}=\pi, \quad \sigma^{2}=\sigma, \quad \pi \sigma=\sigma \pi=0, \quad \pi+\sigma=I, \quad F=\pi-\sigma,
$$

where $\pi$ and $\sigma$ define two complementary distributions [20].

If an almost product manifold $\widetilde{M}$ admits a Lorentzian metric $\widetilde{g}$ such that

$$
\widetilde{g}(F X, F Y)=\widetilde{g}(X, Y),
$$

for any vector fields $X, Y \in \Gamma(T \widetilde{M})$, then $\widetilde{M}=M_{1} \times M_{2}$ is called Lorentzian almost product manifold. From (3.1) and (3.2), we can easily see that

$$
\widetilde{g}(F X, Y)=\widetilde{g}(X, F Y) .
$$

If, for any vector fields $X, Y$ on $\widetilde{M}$,

$$
\left(\stackrel{\widetilde{\nabla}}{X}_{X} F\right) Y=0, \text { that is } \stackrel{\circ}{\nabla}_{X} F Y=F\left(\stackrel{\circ}{\nabla}_{X} Y\right),
$$


then $\widetilde{M}$ is called a Lorentzian product manifold, where $\stackrel{\circ}{\widetilde{\nabla}}$ is the Levi-Civita connection on $\widetilde{M}$ (see, $[20])$.

Now, let $M_{1}$ and $M_{2}$ be real space forms with constant sectional curvatures $c_{1}$ and $c_{2}$ respectively. Then the Riemannian curvature tensor $\stackrel{\circ}{\widetilde{R}}$ of $\widetilde{M}=M_{1}\left(c_{1}\right) \times M_{2}\left(c_{2}\right)$ is given by

$$
\begin{aligned}
\stackrel{\circ}{R}(X, Y) Z= & \frac{1}{16}\left(c_{1}+c_{2}\right)\{\widetilde{g}(Y, Z) X-\widetilde{g}(X, Z) Y \\
& +\widetilde{g}(F Y, Z) F X-\widetilde{g}(F X, Z) F Y\} \\
& +\frac{1}{16}\left(c_{1}-c_{2}\right)\{\widetilde{g}(F Y, Z) X-\widetilde{g}(F X, Z) Y \\
& +\widetilde{g}(Y, Z) F X-\widetilde{g}(X, Z) F Y\},
\end{aligned}
$$

for any $X, Y, Z \in \Gamma(T \widetilde{M})[29]$.

Let $(\widetilde{M}, \widetilde{g}, F)$ be Lorentzian product manifold and $\stackrel{\circ}{\widetilde{\nabla}}$ a Levi-Civita connection on $\widetilde{M}$. A linear connection $\widetilde{\nabla}$ is said to be quarter-symmetric nonmetric connection if the torsion tensor $\widetilde{T}$ is of the form

$$
\widetilde{T}(X, Y)=\widetilde{\pi}(Y) F X-\widetilde{\pi}(X) F Y,
$$

where $\widetilde{\pi}$ is a 1 -form on $\widetilde{M}$ with $\widetilde{Q}$ as associated vector field, that is

$$
\widetilde{g}(\widetilde{Q}, X)=\widetilde{\pi}(X) .
$$

A linear connection $\widetilde{\nabla}$ is called a nonmetric connection if

$$
\left(\widetilde{\nabla}_{X} \widetilde{g}\right)(Y, Z) \neq 0 \text {. }
$$

Let $M$ be a lightlike hypersurface of a Lorentzian product manifold $(\widetilde{M}, \widetilde{g})$. For any $X \in \Gamma(T M)$ we can write

$$
F X=f X+w(X) N,
$$

where $f$ is a $(1,1)$ tensor field and $w$ is a 1 -form on $M$ given by $w(X)=\widetilde{g}(F X, \xi)=$ $\widetilde{g}(X, F \xi)$.

Following [16], a quarter-symmetric non-metric connection $\widetilde{\nabla}$ on $\widetilde{M}$ is given by

$$
\widetilde{\nabla}_{X} Y=\stackrel{\circ}{\nabla}_{X} Y+\widetilde{\pi}(Y) F X,
$$

for any vector fields $X$ and $Y$ of $M$.

From (3.5) the curvature tensor $\widetilde{R}$ of the quarter-symmetric nonmetric connection $\widetilde{\nabla}$ is given by

$$
\widetilde{R}(X, Y) Z=\stackrel{\circ}{\widetilde{R}}(X, Y) Z+\widetilde{\lambda}(X, Z) F Y-\widetilde{\lambda}(Y, Z) F X,
$$


for any vector fields $X, Y \in \Gamma(T M)$, where $\tilde{\lambda}$ is a $(0,2)$ tensor given by $\tilde{\lambda}(X, Z)=$ $\left(\widetilde{\nabla}_{X} \pi\right)((Z)-\pi(Z) \pi(F X)$.

Let $M$ be a lightlike hypersurface of a Lorentzian product manifold $(\widetilde{M}, \widetilde{g})$ with quarter-symmetric nonmetric connection $\widetilde{\nabla}$. Then the Gauss and Weingarten formulas with respect to $\widetilde{\nabla}$ are given by, respectively,

$$
\begin{aligned}
& \widetilde{\nabla}_{X} Y=\nabla_{X} Y+\bar{B}(X, Y) N, \\
& \widetilde{\nabla}_{X} N=-\bar{A}_{N} X+\bar{\tau}(X) N,
\end{aligned}
$$

for any $X, Y \in \Gamma(T M)$.

From (2.4), (3.4), (3.5), (3.7) and (3.8) we obtain

$$
\begin{aligned}
\nabla_{X} Y & =\stackrel{\circ}{\nabla}_{X} Y+\tilde{\pi}(Y) f X, \\
\bar{B}(X, Y) & =B(X, Y)+\widetilde{\pi}(Y) w(X), \\
\bar{A}_{N} X & =A_{N} X-\tilde{\pi}(N) f X, \\
\bar{\tau}(X) & =\tau(X)+\widetilde{\pi}(N) w(X),
\end{aligned}
$$

for any $X, Y \in \Gamma(T M)$.

Using (3.7) we have

$$
R(X, Y, Z, P W)=\widetilde{R}(X, Y, Z, P W)+\bar{B}(Y, Z) \bar{C}(X, P W)-\bar{B}(X, Z) \bar{C}(Y, P W)
$$

for any any $X, Y, Z, W \in \Gamma(T M)$.

From (3.6) and (3.9)

$$
\begin{aligned}
\widetilde{g}(R(X, Y) Z, P W)= & \stackrel{\circ}{g}(\widetilde{R}(X, Y) Z, P W)+\bar{B}(Y, Z) \bar{C}(X, P W)-\bar{B}(X, Z) \bar{C}(Y, P W) \\
& +\widetilde{\lambda}(X, Z) g(F Y, P W)-\widetilde{\lambda}(Y, Z) g(F X, P W),
\end{aligned}
$$

for any any $X, Y, Z, W \in \Gamma(T M)$.

From now on, we will consider a Lorentzian product manifold $\widetilde{M}$ endowed with a quarter-symmetric nonmetric connection $\widetilde{\nabla}$ and the Levi-Civita connection denoted by $\stackrel{\circ}{\widetilde{\nabla}}$.

\section{Chen-Ricci Inequality}

In this section, we use the same notations and terminologies as in [17].

Let $M$ be an $(n+1)$-dimensional lightlike hypersurface of a Lorentzian product manifold $\widetilde{M}=M_{1} \times M_{2}$ with a quarter-symmetric nonmetric connection and $\left\{e_{1}, \ldots, e_{n}, \xi\right\}$ be a basis of $\Gamma(T M)$ where $\left\{e_{1}, \ldots, e_{n}\right\}$ be an orthonormal basis of $\Gamma(S(T M))$ and $n=m_{1}+m_{2}$. For $k \leq n$, we set $\pi_{k, \xi}=\operatorname{Span}\left\{e_{1}, \ldots, e_{k}, \xi\right\}$ is a $(k+1)$ dimensional degenerate plane section and $\pi_{k}=\operatorname{Span}\left\{e_{1}, \ldots, e_{k}\right\}$ is $k$-dimensional non degenerate plane section. Define $k$-degenerate Ricci curvature and $k$-Ricci curvature at a unit 
vector $X \in \Gamma(T M)$ as follows:

$$
\begin{aligned}
\operatorname{Ric}_{\pi_{k, \xi}}(X) & =R^{(0,2)}(X, X)=\sum_{j=1}^{k} g\left(R\left(e_{j}, X\right) X, e_{j}\right)+\widetilde{g}(R(\xi, X) X, N), \\
\operatorname{Ric}_{\pi_{k}}(X) & =R^{(0,2)}(X, X)=\sum_{j=1}^{k} g\left(R\left(e_{j}, X\right) X, e_{j}\right)
\end{aligned}
$$

respectively [17]. Furthermore, $k$-degenerate scalar curvature and $k$-scalar curvature at $p \in M$ are given by

$$
\begin{aligned}
\tau_{\pi_{k, \xi}}(p) & =\sum_{i, j=1}^{k} K_{i j}+\sum_{i=1}^{k} K_{i}^{\text {null }}+K_{i N}, \\
\tau_{\pi_{k}}(p) & =\sum_{i, j=1}^{k} K_{i j},
\end{aligned}
$$

respectively [17]. For $k=n, \pi_{n}=\operatorname{Span}\left\{e_{1}, \ldots, e_{n}\right\}=\Gamma(S(T M))$, we have the screen Ricci curvature and the screen scalar curvature given by

$$
\operatorname{Ric}_{S(T M)}\left(e_{1}\right)=\operatorname{Ric}_{\pi_{n}}\left(e_{1}\right)=\sum_{j=1}^{n} K_{1 j}=K_{12}+\cdots+K_{1 n},
$$

and

$$
\tau_{S(T M)}=\sum_{i, j=1}^{n} K_{i j}
$$

respectively [17].

From (3.3) and (3.10) we can write

$$
\begin{aligned}
\tau_{S(T M)}(p)= & \frac{1}{16}\left(c_{1}+c_{2}\right)\left((i z F)^{2}+n(n-1)\right)+\frac{1}{8}\left(c_{1}-c_{2}\right)(i z F)+\sum_{i, j=1}^{n} m_{i j} \\
& +\sum_{i, j=1}^{n} \bar{B}_{i i} \bar{C}_{j j}-\bar{B}_{i j} \bar{C}_{j i},
\end{aligned}
$$

where $\bar{B}_{i j}=\bar{B}\left(e_{i}, e_{j}\right), \bar{C}_{i j}=\bar{C}\left(e_{i}, e_{j}\right)$ and $m\left(e_{i}, e_{j}\right)=m_{i j}=\widetilde{\lambda}\left(e_{i}, e_{j}\right) g\left(F e_{j}, e_{i}\right)-$ $\widetilde{\lambda}\left(e_{j}, e_{j}\right) g\left(F e_{i}, e_{i}\right)$, for $i, j \in\{1, \ldots, n\}$.

Let $M$ be a screen homothetic lightlike hypersurface of an $(n+2)$-dimensional Lorentzian space form $\widetilde{M}(c)$. Then, from (4.1) we get

$$
\begin{aligned}
\tau_{S(T M)}(p)= & \frac{1}{16}\left(c_{1}+c_{2}\right)\left((i z F)^{2}+n(n-1)\right)+\frac{1}{8}\left(c_{1}-c_{2}\right)(i z F) \\
& +\sum_{i, j=1}^{n} m_{i j}+\varphi n^{2} \mu^{2}-\varphi \sum_{i, j=1}^{n}\left(\bar{B}_{i j}\right)^{2} .
\end{aligned}
$$


Since the sectional curvature of screen homothetic lightlike hypersurface is symmetric, we can denote the screen scalar curvature by $r_{S(T M)}$ as follows:

$$
r_{S(T M)}(p)=\sum_{1 \leq i<j \leq n} K_{i j}=\frac{1}{2} \sum_{i, j=1}^{n} K_{i j}=\frac{1}{2} \tau_{S(T M)}(p) .
$$

By (4.3), (4.2) equality become

$$
\begin{aligned}
2 r_{S(T M)}(p)= & \frac{1}{16}\left(c_{1}+c_{2}\right)\left((i z F)^{2}+n(n-1)\right)+\frac{1}{8}\left(c_{1}-c_{2}\right)(i z F) \\
& +\sum_{i, j=1}^{n} m_{i j}+\varphi n^{2} \mu^{2}-\varphi \sum_{i, j=1}^{n}\left(\bar{B}_{i j}\right)^{2} .
\end{aligned}
$$

Theorem 4.1. Let $M$ be a screen homothetic lightlike hypersurface of a real product space form $\widetilde{M}(c)=M_{1}\left(c_{1}\right) \times M_{2}\left(c_{2}\right)$ of constant sectional curvature $c$, endowed with quarter-symmetric nonmetric connection $\widetilde{\nabla}$. Then, the following statements are true.

(i) For $X \in S^{1}(T M)=\{X \in S(T M):\langle X, X\rangle=1\}$

$$
\begin{aligned}
\operatorname{Ric}_{S(T M)}(X) \leq & \frac{1}{4} \varphi n^{2} \mu^{2}+\frac{1}{32}\left(c_{1}+c_{2}\right)(2(i z F) \bar{g}(F X, X)+3 n-4) \\
& +\frac{1}{16}\left(c_{1}-c_{2}\right)(n-1) \bar{g}(F X, X)-\frac{1}{2} \sum_{2 \leq i<j \leq n} m_{i j} \\
& +\frac{1}{2}\left(\sum_{i=1}^{n} m_{i i}+\sum_{1 \leq j<i \leq n} m_{i j}+\sum_{j=2}^{n} m\left(X, e_{j}\right)\right)
\end{aligned}
$$

(ii) The equality case of (4.5) is satisfied by $X \in T_{p}^{1}(M)$ if and only if

$$
\begin{aligned}
& \bar{B}(X, Y)=0, \quad \text { for all } Y \in T_{p}(M) \text { orthogonal to } X, \\
& \bar{B}(X, X)=\frac{n}{2} \mu .
\end{aligned}
$$

(iii) The equality case of (4.5) holds for all $X \in T_{p}^{1}(M)$ if and only if either $p$ is a totally geodesic point or $n=2$ and $p$ is a totally umbilical point.

Proof. From (4.4) we get

$$
\begin{aligned}
\frac{1}{4} \varphi n^{2} \mu^{2}= & r_{S(T M)}(p)-\frac{1}{32}\left(c_{1}+c_{2}\right)\left((i z F)^{2}+n(n-1)\right)-\frac{1}{16}\left(c_{1}-c_{2}\right)(i z F) \\
& -\frac{1}{2} \sum_{i, j=1}^{n} m_{i j}+\frac{1}{4} \varphi\left(\bar{B}_{11}-\bar{B}_{22}-\cdots-\bar{B}_{n n}\right)^{2}+\varphi \sum_{j=2}^{n}\left(\bar{B}_{1 j}\right)^{2} \\
& -\varphi \sum_{2 \leq i<j \leq n}^{m}\left(\bar{B}_{i i} \bar{B}_{j j}-\left(\bar{B}_{i j}\right)^{2}\right)
\end{aligned}
$$


Using (3.3) and (3.10) we also have

$$
\begin{aligned}
\varphi \sum_{2 \leq i<j \leq n}^{m}\left(\bar{B}_{i i} \bar{B}_{j j}-\left(\bar{B}_{i j}\right)^{2}\right)= & \sum_{2 \leq i<j \leq n} K_{i j}-\sum_{2 \leq i<j \leq n} \widetilde{K}_{i j} \\
= & \sum_{2 \leq i<j \leq n} K_{i j}-\frac{1}{32}\left(c_{1}+c_{2}\right)\left((i z F)^{2}-2(i z F) \bar{g}\left(F e_{1}, e_{1}\right)\right) \\
& -\frac{1}{16}\left(c_{1}-c_{2}\right)\left((i z F)-(n-1) \bar{g}\left(F e_{1}, e_{1}\right)\right) \\
& -\frac{1}{32}\left(c_{1}+c_{2}\right)(n-2)^{2}-\sum_{2 \leq i<j \leq n} m_{i j} .
\end{aligned}
$$

From (4.7) and (4.8) we obtain

$$
\begin{aligned}
\operatorname{Ric}_{S(T M)}\left(e_{1}\right)= & \frac{1}{4} \varphi n^{2} \mu^{2} \varphi-\frac{1}{4} \varphi\left(\bar{B}_{11}-\bar{B}_{22}-\cdots-\bar{B}_{n n}\right)^{2}-\varphi \sum_{j=2}^{n}\left(\bar{B}_{1 j}\right)^{2} \\
& +\frac{1}{32}\left(c_{1}+c_{2}\right)\left(2(i z F) \bar{g}\left(F e_{1}, e_{1}\right)+3 n-4\right)-\sum_{2 \leq i<j \leq n} m_{i j} \\
& +\frac{1}{16}\left(c_{1}-c_{2}\right)(n-1) \bar{g}\left(F e_{1}, e_{1}\right) \\
& +\frac{1}{2}\left(\sum_{i=1}^{n} m_{i i}+\sum_{1 \leq j<i \leq n} m_{i j}+\sum_{j=2}^{n} m_{1 j}\right) .
\end{aligned}
$$

If we put $e_{1}=X$ as any vector of $T_{p}^{1}(M)$ in (4.9) we obtain (4.5).

The equality case of (4.5) holds for $X \in T_{p}^{1}(M)$ if and only if

$$
\bar{B}_{12}=\bar{B}_{13}=\cdots=\bar{B}_{1 n}=0 \text { and } \bar{B}_{11}=\bar{B}_{22}+\cdots+B_{n n},
$$

equivalent to (4.6).

Now we prove the statement (iii). Assuming the equality case of (4.5) for all $X \in T_{p}^{1}(M)$, in view of (4.10), we have

$$
\bar{B}_{i j}=0, \quad i \neq j
$$

and

$$
2 \bar{B}_{i i}=\bar{B}_{11}+\bar{B}_{22}+\cdots+\bar{B}_{n n}, \quad i \in\{1, \ldots, n\} .
$$

From (4.12) we have $2 \bar{B}_{11}=2 \bar{B}_{22}=\cdots=2 \bar{B}_{n n}=\bar{B}_{11}+\bar{B}_{22}+\cdots+\bar{B}_{n n}$ which implies that

$$
(n-2)\left(\bar{B}_{11}+\bar{B}_{22}+\cdots+\bar{B}_{n n}\right)=0 .
$$

Thus, either $\bar{B}_{11}+\bar{B}_{22}+\cdots+\bar{B}_{n n}=0$ or $n=2$. If $\bar{B}_{11}+\bar{B}_{22}+\cdots+\bar{B}_{n n}=0$, then in view of (4.12), we get $\bar{B}_{i i}=0$ for all $i \in\{1, \ldots, n\}$. This together with (4.11) gives $\bar{B}_{i j}=0$ for all $i, j \in\{1, \ldots, n\}$, that is, $p$ is a totally geodesic point. If $n=2$, then 
from (4.12), $2 \bar{B}_{11}=2 \bar{B}_{22}=\bar{B}_{11}+\bar{B}_{22}$, which shows that $p$ is a totally umbilical point. The proof of the converse part is straightforward.

We recall the following algebraic Lemma from [27].

Lemma 4.1. Let $a_{1}, a_{2}, \ldots, a_{n}$, be $n$-real number $(n>1)$, then

$$
\frac{1}{n}\left(\sum_{i=1}^{n} a_{i}\right)^{2} \leq \sum_{i=1}^{n} a_{i}^{2}
$$

with equality if and only if $a_{1}=a_{2}=\cdots=a_{n}$.

Theorem 4.2. Let $M$ be a screen homothetic lightlike hypersurface of a real product space form $\widetilde{M}(c)=M_{1}\left(c_{1}\right) \times M_{2}\left(c_{2}\right)$ of constant sectional curvature $c$, endowed with quarter-symmetric nonmetric connection $\widetilde{\nabla}$

$$
\begin{aligned}
\tau_{S(T M)}(p) \leq & \varphi n(n-1) \mu^{2}+\frac{1}{16}\left(c_{1}+c_{2}\right)\left((i z F)^{2}+n(n-1)\right) \\
& +\frac{1}{8}\left(c_{1}-c_{2}\right)(i z F)+\sum_{i, j=1}^{n} m_{i j}
\end{aligned}
$$

with equality if and only if $p$ is a totally umbilical point.

Proof. From (4.2) we have

$$
\begin{aligned}
\varphi n^{2} \mu^{2}= & \tau_{S(T M)}(p)+\varphi \sum_{i=1}^{n}\left(B_{i i}\right)^{2}+\varphi \sum_{i \neq j}\left(B_{i j}\right)^{2}-\sum_{i, j=1}^{n} m_{i j} \\
& -\frac{1}{16}\left(c_{1}+c_{2}\right)\left((i z F)^{2}+n(n-1)\right)-\frac{1}{8}\left(c_{1}-c_{2}\right)(i z F) .
\end{aligned}
$$

Using Lemma 4.1 we get

$$
n \mu^{2} \leq \sum_{i=1}^{n}\left(B_{i i}\right)^{2} .
$$

Considering (4.14) and (4.15) we obtain (4.13). Equality case of (4.13) holds if and only if

$$
\bar{B}_{11}=\bar{B}_{22}=\cdots=\bar{B}_{n n},
$$

the shape operator $A_{\xi}^{*}$ take the form:

$$
A_{\xi}^{*}=\left[\begin{array}{ccccc}
\bar{B}_{11} & 0 & \cdots & 0 & 0 \\
0 & \bar{B}_{11} & \cdots & 0 & 0 \\
\vdots & \vdots & \ddots & \vdots & \vdots \\
0 & 0 & \cdots & \bar{B}_{11} & 0 \\
0 & 0 & \cdots & 0 & 0
\end{array}\right]
$$

which shows that $M$ is totally umbilical. This completes the proof of the theorem. 
Also, the components of the second fundamental form $\bar{B}$ and the screen second fundamental form $\bar{C}$ satisfy

$$
\sum_{i, j=1}^{n} \bar{B}_{i j} \bar{C}_{j i}=\frac{1}{2}\left\{\sum_{i, j=1}^{n}\left(\bar{B}_{i j}+\bar{C}_{j i}\right)^{2}-\sum_{i, j=1}^{n}\left(\bar{B}_{i j}\right)^{2}+\left(\bar{C}_{j i}\right)^{2}\right\},
$$

and

$$
\sum_{i, j} \bar{B}_{i i} \bar{C}_{j j}=\frac{1}{2}\left\{\left(\sum_{i, j} \bar{B}_{i i}+\bar{C}_{j j}\right)^{2}-\left(\sum_{i} \bar{B}_{i i}\right)^{2}-\left(\sum_{j} C_{j j}\right)^{2}\right\}
$$

Theorem 4.3. Let $M$ be lightlike hypersurface of a real product space form $\widetilde{M}(c)=$ $M_{1}\left(c_{1}\right) \times M_{2}\left(c_{2}\right)$ of constant sectional curvature $c$, endowed with quarter-symmetric nonmetric connection $\widetilde{\nabla}$. Then

(i)

$$
\begin{aligned}
\tau_{S(T M)}(p) \leq & n \mu \operatorname{trace} A_{N}+\frac{1}{2} \sum_{i, j=1}^{n}\left(\left(\bar{B}_{i j}\right)^{2}+\left(\bar{C}_{j i}\right)^{2}\right)+\sum_{i, j=1}^{n} m_{i j} \\
& +\frac{1}{16}\left(c_{1}+c_{2}\right)\left((i z F)^{2}+n(n-1)\right)+\frac{1}{8}\left(c_{1}-c_{2}\right)(i z F) .
\end{aligned}
$$

The equality case of (4.19) holds for all $p \in M$ if and only if either $M$ is a screen homothetic lightlike hypersurface with $\varphi=-1$ or $M$ is a totally geodesic lightlike hypersurface.

(ii)

$$
\begin{aligned}
\tau_{S(T M)}(p) \geq & n \mu \operatorname{trace} A_{N}-\frac{1}{2} \sum_{i, j=1}^{n}\left(\left(\bar{B}_{i j}\right)^{2}+\left(\bar{C}_{j i}\right)^{2}\right)+\sum_{i, j=1}^{n} m_{i j} \\
& +\frac{1}{16}\left(c_{1}+c_{2}\right)\left((i z F)^{2}+n(n-1)\right)+\frac{1}{8}\left(c_{1}-c_{2}\right)(i z F) .
\end{aligned}
$$

The equality case of (4.20) holds for all $p \in M$ if and only if either $M$ is a screen homothetic lightlike hypersurface with $\varphi=1$ or $M$ is a totally geodesic lightlike hypersurface.

(iii) The equalities case of (4.19) and (4.20) hold at $p \in M$ if and only if $p$ is a totally geodesic point.

Proof. Using (4.1) and (4.17), we get

$$
\begin{aligned}
\tau_{S(T M)}(p)= & \sum_{i, j=1}^{n} \bar{B}_{i i} \bar{C}_{j j}-\frac{1}{2} \sum_{i, j=1}^{n}\left(\bar{B}_{i j}+\bar{C}_{j i}\right)+\frac{1}{2} \sum_{i, j=1}^{n}\left(\left(\bar{B}_{i j}\right)^{2}+\left(\bar{C}_{j i}\right)^{2}\right) \\
& +\frac{1}{16}\left(c_{1}+c_{2}\right)\left((i z F)^{2}+n(n-1)\right)+\frac{1}{8}\left(c_{1}-c_{2}\right)(i z F)+\sum_{i, j=1}^{n} m_{i j},
\end{aligned}
$$

which yields (4.19). 
Since

$$
\frac{1}{2}\left(\left(\bar{B}_{i j}\right)^{2}+\left(\bar{C}_{j i}\right)^{2}\right)=\frac{1}{4}\left(\bar{B}_{i j}+\bar{C}_{j i}\right)^{2}+\frac{1}{4}\left(\bar{B}_{i j}-\bar{C}_{j i}\right)^{2}
$$

we obtain

$$
\begin{aligned}
\tau_{S(T M)}(p)= & \sum_{i, j=1}^{n} \bar{B}_{i i} C_{j j}-\frac{1}{2} \sum_{i, j=1}^{n}\left(\left(\bar{B}_{i j}\right)^{2}+\left(\bar{C}_{j i}\right)^{2}\right)+\frac{1}{2} \sum_{i, j=1}^{n}\left(\bar{B}_{i j}-\bar{C}_{j i}\right)^{2} \\
& +\frac{1}{16}\left(c_{1}+c_{2}\right)\left((i z F)^{2}+n(n-1)\right)+\frac{1}{8}\left(c_{1}-c_{2}\right)(i z F)+\sum_{i, j=1}^{n} m_{i j}
\end{aligned}
$$

which yields (4.20). From (4.19), (4.20), (4.21) and (4.23) it is easy to get (i), (ii) and (iii) statements.

By Theorem 4.3 we have the following corollary.

Corollary 4.1. Let $M$ be a screen homothetic lightlike hypersurface of a real product space form $\widetilde{M}(c)=M_{1}\left(c_{1}\right) \times M_{2}\left(c_{2}\right)$ of constant sectional curvature $c$, endowed with quarter-symmetric nonmetric connection $\widetilde{\nabla}$. Then, we have

$$
\begin{aligned}
\tau_{S(T M)}(p) \leq & \varphi n^{2} \mu^{2}+\frac{\left(1+\varphi^{2}\right)}{2} \sum_{i, j=1}^{n}\left(\bar{B}_{i j}\right)^{2}+\frac{1}{16}\left(c_{1}+c_{2}\right)\left((i z F)^{2}+n(n-1)\right) \\
& +\frac{1}{8}\left(c_{1}-c_{2}\right)(i z F)+\sum_{i, j=1}^{n} m_{i j} .
\end{aligned}
$$

and

$$
\begin{aligned}
\tau_{S(T M)}(p) \geq & \varphi n^{2} \mu^{2}-\frac{\left(1+\varphi^{2}\right)}{2} \sum_{i, j=1}^{n}\left(\bar{B}_{i j}\right)^{2}+\frac{1}{16}\left(c_{1}+c_{2}\right)\left((i z F)^{2}+n(n-1)\right) \\
& +\frac{1}{8}\left(c_{1}-c_{2}\right)(i z F)+\sum_{i, j=1}^{n} m_{i j} .
\end{aligned}
$$

Theorem 4.4. Let $M$ be lightlike hypersurface of a real product space form $\widetilde{M}(c)=$ $M_{1}\left(c_{1}\right) \times M_{2}\left(c_{2}\right)$ of constant sectional curvature $c$, endowed with quarter-symmetric nonmetric connection $\widetilde{\nabla}$. Then, we have

$$
\begin{aligned}
\tau_{S(T M)}(p) \leq & \frac{1}{2}(\operatorname{trace} \bar{A})^{2}-\frac{1}{2}\left(\operatorname{trace} A_{N}\right)^{2}-\frac{1}{4} \sum_{i, j=1}^{n}\left(\bar{B}_{i j}+\bar{C}_{j i}\right)^{2} \\
& +\frac{1}{4} \sum_{i, j=1}^{n}\left(\bar{B}_{i j}-\bar{C}_{j i}\right)^{2}+\frac{1}{16}\left(c_{1}+c_{2}\right)\left((i z F)^{2}+n(n-1)\right) \\
& +\frac{1}{8}\left(c_{1}-c_{2}\right)(i z F)+\sum_{i, j=1}^{n} m_{i j},
\end{aligned}
$$


where

$$
\bar{A}=\left[\begin{array}{cccc}
\bar{B}_{11}+\bar{C}_{11} & \bar{B}_{12}+\bar{C}_{21} & \cdots & \bar{B}_{1 n}+\bar{C}_{n 1} \\
\bar{B}_{21}+\bar{C}_{12} & \bar{B}_{22}+\bar{C}_{22} & \cdots & \bar{B}_{2 n}+\bar{C}_{n 2} \\
\vdots & \vdots & \ddots & \vdots \\
\bar{B}_{n 1}+C_{1 n} & \bar{B}_{n 2}+\bar{C}_{2 n} & \cdots & \bar{B}_{n n}+\bar{C}_{n n}
\end{array}\right]
$$

The equality case of (4.24) holds for all $p \in M$ if and only if $M$ is minimal.

Proof. From (4.1), (4.17) and (4.18) we get

$$
\begin{aligned}
\tau_{S(T M)}(p)= & \frac{1}{2}\left(\sum_{i, j} \bar{B}_{i i}+C_{j j}\right)^{2}-\frac{1}{2}\left(\sum_{i} \bar{B}_{i i}\right)^{2}-\frac{1}{2}\left(\sum_{j} C_{j j}\right)^{2} \\
& -\frac{1}{2} \sum_{i, j=1}^{n}\left(\bar{B}_{i j}+\bar{C}_{j i}\right)^{2}+\frac{1}{2} \sum_{i, j=1}^{n}\left(\left(\bar{B}_{i j}\right)^{2}+\left(\bar{C}_{j i}\right)^{2}\right)+\sum_{i, j=1}^{n} m_{i j} \\
& +\frac{1}{16}\left(c_{1}+c_{2}\right)\left((i z F)^{2}+n(n-1)\right)+\frac{1}{8}\left(c_{1}-c_{2}\right)(i z F) .
\end{aligned}
$$

From (4.22) we have

$$
\begin{aligned}
& -\frac{1}{2} \sum_{i, j=1}^{n}\left(\bar{B}_{i j}+\bar{C}_{j i}\right)^{2}+\frac{1}{2} \sum_{i, j=1}^{n}\left(\bar{B}_{i j}\right)^{2}+\left(\bar{C}_{j i}\right)^{2} \\
= & -\frac{1}{4} \sum_{i, j=1}^{n}\left(\bar{B}_{i j}+\bar{C}_{j i}\right)^{2}+\frac{1}{4} \sum_{i, j=1}^{n}\left(\bar{B}_{i j}-\bar{C}_{j i}\right)^{2} .
\end{aligned}
$$

If we put (4.27) in (4.26), we obtain

$$
\begin{aligned}
\tau_{S(T M)}(p)= & \frac{1}{2}\left(\sum_{i, j} \bar{B}_{i i}+C_{j j}\right)^{2}-\frac{1}{2}\left(\sum_{i} \bar{B}_{i i}\right)^{2}-\frac{1}{2}\left(\sum_{j} C_{j j}\right)^{2} \\
& -\frac{1}{4} \sum_{i, j=1}^{n}\left(\bar{B}_{i j}+\bar{C}_{j i}\right)^{2}+\frac{1}{4} \sum_{i, j=1}^{n}\left(\bar{B}_{i j}-\bar{C}_{j i}\right)^{2}+\sum_{i, j=1}^{n} m_{i j} \\
& +\frac{1}{16}\left(c_{1}+c_{2}\right)\left((i z F)^{2}+n(n-1)\right)+\frac{1}{8}\left(c_{1}-c_{2}\right)(i z F) .
\end{aligned}
$$

The equality case of (4.24) satisfies then

$$
\sum_{i} \bar{B}_{i i}=0
$$

This shows that $M$ is minimal.

By Theorem 4.4 we have the following corollary. 
Corollary 4.2. Let $M$ be a screen homothetic lightlike hypersurface of a real product space form $\widetilde{M}(c)=M_{1}\left(c_{1}\right) \times M_{2}\left(c_{2}\right)$ of constant sectional curvature $c$, endowed with quarter-symmetric nonmetric connection $\widetilde{\nabla}$

$$
\begin{aligned}
\tau_{S(T M)}(p) \leq & \frac{(2 \varphi+1)}{2} n^{2} \mu^{2}-\varphi \sum_{i, j=1}^{n}\left(\bar{B}_{i j}\right)^{2}+\frac{1}{16}\left(c_{1}+c_{2}\right)\left((i z F)^{2}+n(n-1)\right) \\
& +\frac{1}{8}\left(c_{1}-c_{2}\right)(i z F)+\sum_{i, j=1}^{n} m_{i j} .
\end{aligned}
$$

The equality case of (4.28) holds for all $p \in M$ if and only if $M$ is minimal.

Theorem 4.5. Let $M$ be lightlike hypersurface of a real product space form $\widetilde{M}(c)=$ $M_{1}\left(c_{1}\right) \times M_{2}\left(c_{2}\right)$ of constant sectional curvature $c$, endowed with quarter-symmetric nonmetric connection $\widetilde{\nabla}$. Then, we have

$$
\begin{aligned}
\tau_{S(T M)}(p) \leq & \frac{n-1}{2 n}(\operatorname{trace} \bar{A})^{2}-\frac{1}{2}\left(\operatorname{trace} A_{N}\right)^{2}-\frac{1}{2} n^{2} \mu^{2}-\frac{1}{2} \sum_{i \neq j}\left(\bar{B}_{i j}+\bar{C}_{j i}\right)^{2} \\
& +\frac{1}{2} \sum_{i, j=1}^{n}\left(\left(\bar{B}_{i j}\right)^{2}+\left(\bar{C}_{j i}\right)^{2}\right)+\frac{1}{16}\left(c_{1}+c_{2}\right)\left((i z F)^{2}+n(n-1)\right) \\
& +\frac{1}{8}\left(c_{1}-c_{2}\right)(i z F)+\sum_{i, j=1}^{n} m_{i j},
\end{aligned}
$$

where $\bar{A}$ is equal to $(4.25)$.

The equality case of (4.29) holds for all $p \in M$ if and only if $n \mu=-\operatorname{trace} A_{N}$.

Proof. From (4.26)

$$
\begin{aligned}
\tau_{S(T M)}(p)= & \frac{1}{2}(\operatorname{trace} \bar{A})^{2}-\frac{1}{2}\left(\operatorname{trace} A_{N}\right)^{2}-\frac{1}{2} n^{2} \mu^{2}-\frac{1}{2} \sum_{i}\left(\bar{B}_{i i}+\bar{C}_{i i}\right)^{2} \\
& -\frac{1}{2} \sum_{i \neq j}\left(\bar{B}_{i j}+\bar{C}_{j i}\right)^{2}+\frac{1}{2} \sum_{i, j=1}^{n}\left(\left(\bar{B}_{i j}\right)^{2}+\left(\bar{C}_{j i}\right)^{2}\right)+\sum_{i, j=1}^{n} m_{i j} \\
& +\frac{1}{16}\left(c_{1}+c_{2}\right)\left((i z F)^{2}+n(n-1)\right)+\frac{1}{8}\left(c_{1}-c_{2}\right)(i z F) .
\end{aligned}
$$

Using Lemma 4.1 and equality case of (4.30), we have

$$
\begin{aligned}
\tau_{S(T M)}(p) \leq & \frac{1}{2}(\operatorname{trace} \bar{A})^{2}-\frac{1}{2}\left(\operatorname{trace} A_{N}\right)^{2}-\frac{1}{2} n^{2} \mu^{2}-\frac{1}{2 n} \sum_{i}\left(\bar{B}_{i i}+\bar{C}_{i i}\right)^{2} \\
& -\frac{1}{2} \sum_{i \neq j}\left(\bar{B}_{i j}+\bar{C}_{j i}\right)^{2}+\frac{1}{2} \sum_{i, j=1}^{n}\left(\bar{B}_{i j}\right)^{2}+\left(\bar{C}_{j i}\right)^{2}+\sum_{i, j=1}^{n} m_{i j} \\
& +\frac{1}{16}\left(c_{1}+c_{2}\right)\left((i z F)^{2}+n(n-1)\right)+\frac{1}{8}\left(c_{1}-c_{2}\right)(i z F),
\end{aligned}
$$


which implies (4.29). The equality case of (4.29) holds, then

$$
\bar{B}_{11}+\bar{C}_{11}=\cdots=\bar{B}_{n n}+\bar{C}_{n n} .
$$

From (4.31) we get

$$
\begin{gathered}
(1-n) \bar{B}_{11}+\bar{B}_{22}+\cdots+\bar{B}_{n n}+(1-n) \bar{C}_{11}+\bar{C}_{22}+\cdots+\bar{C}_{n n}=0 \\
\bar{B}_{11}+(1-n) \bar{B}_{22}+\cdots+\bar{B}_{n n}+\bar{C}_{11}+(1-n) \bar{C}_{22}+\cdots+\bar{C}_{n n}=0 \\
\vdots \\
\bar{B}_{11}+\bar{B}_{22}+\cdots+(1-n) \bar{B}_{n n}+\bar{C}_{11}+\bar{C}_{22}+\cdots+(1-n) \bar{C}_{n n}=0
\end{gathered}
$$

By the above equations, we have

$$
(n-1)^{2}\left(\operatorname{trace} A_{N}+n \mu\right)=0 .
$$

Since $n \neq 1$, we obtain $n \mu=-\operatorname{trace} A_{N}$.

By Theorem 4.5 we have the following corollary.

Corollary 4.3. Let $M$ be screen homothetic lightlike hypersurface of a real product space form $\widetilde{M}(c)=M_{1}\left(c_{1}\right) \times M_{2}\left(c_{2}\right)$ of constant sectional curvature $c$, endowed with quarter-symmetric nonmetric connection $\widetilde{\nabla}$. Then

$$
\begin{aligned}
& \tau_{S(T M)}(p) \leq \varphi n(n-1) \mu^{2}-\frac{\left(1+\varphi^{2}\right)}{2} n \mu^{2}-\frac{(1+\varphi)^{2}}{2} \sum_{i \neq j}\left(\bar{B}_{i j}\right)^{2}+\frac{\left(1+\varphi^{2}\right)}{2} \sum_{i, j=1}^{n}\left(\bar{B}_{i j}\right)^{2} \\
&(4.32)+\frac{1}{16}\left(c_{1}+c_{2}\right)\left((i z F)^{2}+n(n-1)\right)+\frac{1}{8}\left(c_{1}-c_{2}\right)(i z F)+\sum_{i, j=1}^{n} m_{i j} .
\end{aligned}
$$

The equality case of (4.32) holds for all $p \in M$ if and only if either $\varphi=-1$ or $M$ is minimal.

Theorem 4.6. Let $M$ be lightlike hypersurface of a real product space form $\widetilde{M}(c)=$ $M_{1}\left(c_{1}\right) \times M_{2}\left(c_{2}\right)$ of constant sectional curvature $c$, endowed with quarter-symmetric nonmetric connection $\widetilde{\nabla}$. Then

$$
\begin{aligned}
\tau_{S(T M)}(p) \geq & \frac{1}{2}(\operatorname{trace} \bar{A})^{2}-\frac{1}{2}\left(\operatorname{trace} A_{N}\right)^{2}-\frac{1}{2} n(n-1) \mu^{2}-\frac{1}{2} \sum_{i, j=1}^{n}\left(\bar{B}_{i j}+\bar{C}_{j i}\right)^{2} \\
& +\frac{1}{2} \sum_{i \neq j}\left(\bar{B}_{i j}\right)^{2}+\frac{1}{2} \sum_{i, j=1}^{n}\left(\bar{C}_{j i}\right)^{2}+\frac{1}{16}\left(c_{1}+c_{2}\right)\left((i z F)^{2}+n(n-1)\right) \\
& +\frac{1}{8}\left(c_{1}-c_{2}\right)(i z F)+\sum_{i, j=1}^{n} m_{i j} .
\end{aligned}
$$

The equality case of (4.33) holds for all $p \in M$ if and only if $p$ is a totally umbilical point. 
Proof. From (4.26)

$$
\begin{aligned}
\tau_{S(T M)}(p)= & \frac{1}{2}(\operatorname{trace} \bar{A})^{2}-\frac{1}{2}\left(\operatorname{trace} A_{N}\right)^{2}-\frac{1}{2} n^{2} \mu^{2}+\frac{1}{2} \sum_{i}\left(\bar{B}_{i i}\right)^{2}+\frac{1}{2} \sum_{i \neq j}\left(\bar{B}_{i j}\right)^{2} \\
& +\frac{1}{2} \sum_{i, j=1}^{n}\left(\bar{C}_{j i}\right)^{2}-\frac{1}{2} \sum_{i, j=1}^{n}\left(\bar{B}_{i j}+\bar{C}_{j i}\right)^{2}+\sum_{i, j=1}^{n} m_{i j} \\
& +\frac{1}{16}\left(c_{1}+c_{2}\right)\left((i z F)^{2}+n(n-1)\right)+\frac{1}{8}\left(c_{1}-c_{2}\right)(i z F) .
\end{aligned}
$$

Using Lemma 4.1 and equality case of (4.34) we have

$$
\begin{aligned}
\tau_{S(T M)}(p) \geq & \frac{1}{2}(\operatorname{trace} \bar{A})^{2}-\frac{1}{2}\left(\operatorname{trace} A_{N}\right)^{2}-\frac{1}{2} n^{2} \mu^{2}+\frac{1}{2 n}\left(\sum_{i} \bar{B}_{i i}\right)^{2} \\
& +\frac{1}{2} \sum_{i \neq j}\left(\bar{B}_{i j}\right)^{2}+\frac{1}{2} \sum_{i, j=1}^{n}\left(\bar{C}_{j i}\right)^{2}-\frac{1}{2} \sum_{i, j=1}^{n}\left(\bar{B}_{i j}+\bar{C}_{j i}\right)^{2}+\sum_{i, j=1}^{n} m_{i j} \\
& +\frac{1}{16}\left(c_{1}+c_{2}\right)\left((i z F)^{2}+n(n-1)\right)+\frac{1}{8}\left(c_{1}-c_{2}\right)(i z F),
\end{aligned}
$$

which implies (4.33). Equality case of (4.33) holds if and only if $\bar{B}_{11}=\cdots=\bar{B}_{n n}$ the shape operator $A_{\xi}^{*}$ take the form as (4.16), which shows that $M$ is totally umbilical. This completes the proof of the theorem.

By Theorem 4.6 we have the following corollary.

Corollary 4.4. Let $M$ be screen homothetic lightlike hypersurface of a real product space form $\widetilde{M}(c)=M_{1}\left(c_{1}\right) \times M_{2}\left(c_{2}\right)$ of constant sectional curvature $c$, endowed with quarter-symmetric nonmetric connection $\widetilde{\nabla}$. Then

$$
\begin{aligned}
\tau_{S(T M)}(p) \geq & \frac{(2 \varphi+1)}{2} n^{2} \mu^{2}-\frac{1}{2} n(n-1) \mu^{2}-\frac{(2 \varphi+1)}{2} \sum_{i, j=1}^{n}\left(\bar{B}_{i j}\right)^{2} \\
& +\frac{1}{16}\left(c_{1}+c_{2}\right)\left((i z F)^{2}+n(n-1)\right)+\frac{1}{8}\left(c_{1}-c_{2}\right)(i z F)+\sum_{i, j=1}^{n} m_{i j} .
\end{aligned}
$$

The equality case of (4.35) holds for all $p \in M$ if and only if $p$ is a totally umbilical point.

\section{REFERENCES}

[1] M. Atçeken, Submanifolds of Riemannian product manifolds, Turkish J. Math. 29 (2005), 389401.

[2] M. Atçeken and E. Kılıç, Semi-invariant lightlike submanifolds of a semi-Riemannian product manifold, Kodai Math. J. 30(3) (2007), 361-378.

[3] C. Atindogbe and K. L. Duggal, Conformal screen on lightlike hypersurfaces, Int. J. Pure Appl. Math., 11(4) (2004), 421-442. 
[4] J. K. Beem, P. E. Ehrlich and K. L. Easley, Global Lorentzian Geometry, $2^{\text {nd }}$ Edition, Marcel Dekker, Inc., New York, 1996.

[5] C. L. Bejan and K. L. Duggal, Global lightlike manifolds and harmonicity, Kodai Math. J. 28 (2005), 131-145.

[6] R. L. Bishop and B. O'Neill, Manifolds of negative curvature, Trans. Amer. Math. Soc. 145 (1969),1-49.

[7] B. Y. Chen, Mean curvature and shape operator of isometric immersion in real space forms, Glasg. Math. J. 38 (1996), 87-97.

[8] B. Y. Chen, Relation between Ricci curvature and shape operator for submanifolds with arbitrary codimension, Glasg. Math. J. 41 (1999), 33-41.

[9] B. Y. Chen, Some pinching and classification theorems for minimal submanifolds, Arch. Math. (Basel) 60(6) (1993), 568-578.

[10] B. Y. Chen, A Riemannian invariant and its applications to submanifold theory, Result Math. 27 (1995), 17-26.

[11] B. Y. Chen, F. Dillen, L. Verstraelen and V. Vrancken, Characterizations of Riemannian space forms, Einstein spaces and conformally flat spaces, Proc. Amer. Math. Soc. 128 (2000), 589-598.

[12] B. Y. Chen, A Riemannian invariant for submanifolds in space forms and its applications, Geometry and Topology of Submanifolds VI (1994), 58-81.

[13] B. Y. Chen, Pseudo-Riemannian Geometry, $\delta$-Invariants and Applications, World Scientific, Singapore, 2011.

[14] K. L. Duggal and A. Bejancu, Lightlike Submanifold of Semi-Riemannian Manifolds and Applications, Kluwer Academic Pub., The Netherlands, 1996.

[15] K. L. Duggal and B. Şahin, Differential Geometry of Lightlike Submanifolds, Birkhäuser Verlag AG., 2010.

[16] S. Golab, On semi-symmetric and quarter-symmetric linear connection, Tensor (N.S.) 29 (1975), 249-254.

[17] M. Gülbahar, E. Kılıç and S. Keleş, Chen-like inequalities on lightlike hypersurfaces of a Lorentzian manifold, J. Inequal. Appl. 2013, Article ID 266.

[18] M. Gülbahar, M., E. Kılı̧ and S. Keleş, Some inequalities on screen homothetic lightlike hypersurfaces of a Lorentzian manifold, Taiwanese J. Math. 17(6) (2013), 2083-2100.

[19] D. Kamilya and U. C. De, Some properties of a Ricci quarter-symmetric metric connection in a Riemanian manifold, Indian J. Pure and Appl. Math. 26(1) (1995), 29-34.

[20] E. Kılıç and B. Oğuzhan, Lightlike hypersurfaces of a semi-Riemannian product manifold and quarter-symmetric nonmetric connections, Int. J. Math. Math. Sci. 2012, Article ID 178390.

[21] X. Liu, and J. Zhou, On Ricci curvature of certain submanifolds in cosympletic space form, Sarajeva J. Math. 2(1) (2006), 95-106.

[22] A. Mihai and C. Özgür, Chen inequalities for submanifolds of real space form with a semisymmetric metric connection, Taiwanese J. Math. 14(4) (2010), 1465-1477.

[23] B. O'Neill, Semi-Riemannian Geometry with Applications to Relativity, Academic Press, London, 1983.

[24] S. C. Rastogi, On quarter-symmetric metric connection, C. R. Acad. Bulgare Sci. 31(7) (1978), 811-814.

[25] X. Senlin and N. Yilong, Submanifold of product Riemannian manifold, Acta Math. Sci. 20(2) (2000), 213-218.

[26] B. Şahin and M. Atçeken, Semi-invariant submanifolds of Riemannian product manifold. Balkan J. Geom. Appl. 8(1) (2003), 91-100.

[27] M. M. Tripathi, Certain basic inequalities for submanifolds in $(\kappa, \mu)$ space, in: K. L. Duggal and R. Sharma (Eds.), Proceedings of the AMS Special Session on Recent Advances in Riemannian and Lorentzian Geometries, Baltimore, USA, 2003, pp. 187-202. 
[28] M. M. Tripathi, Improved Chen-Ricci inequality for curvature-like tensor and its applications, Differential Geom. Appl. 28 (2011), 685-698.

[29] K. Yano and M. Kon, Structure on Manifolds, World Scientific Publishing Co. Ltd, 1984.

${ }^{1}$ Department of Mathematics, ÇUKUROVA UNIVERSITY,

ADANA, TURKEY

E-mail address: nonen@cu.edu.tr

${ }^{2}$ Department of Mathematics, MERsin UnIVERSITY,

Mersin, Turkey

E-mail address: yerol@mersin.edu.tr 